\title{
New fiber-reactive UV-absorbers increasing protective properties of cellulose fibres
}

\author{
Justyna Mamnicka • Wojciech Czajkowski
}

Received: 3 February 2012/ Accepted: 23 July 2012/Published online: 2 August 2012

(C) The Author(s) 2012. This article is published with open access at Springerlink.com

\begin{abstract}
Several new UV-absorbers with built-in monochlorotriazine and vinylsulphone cellulose-reactive residues were synthesised. Their application properties on various cellulose-based textile materials were determined and the UV-protection factor values of modified fabrics were measured.
\end{abstract}

Keywords Reactive UV-absorbers for cellulose fibres · Ultraviolet protection factor (UPF) .

Bamboo viscose

\section{Introduction}

In recent years the protection against sunlight ultraviolet radiation is of high practical interest. Sunlight radiation traditionally is divided into three main regions, ultraviolet, visible and infrared. Ultraviolet radiation is the most dangerous for living species and other organic materials because it is characterised by the highest energy per photon which exceeds the energy of carbon-carbon single bond (Reinert et al. 1997). Prolonged action of UV radiation results in many adverse effects. In synthetic materials it can be observed changes of their physical and mechanical

J. Mamnicka $(\bowtie) \cdot$ W. Czajkowski

Institute of Polymer and Dye Technology, Technical

University of Lodz, ul. Stefanowskiego 12/16, 90-924

Lodz, Poland

e-mail: justyna.mamnicka@gmail.com properties such as loss of colour, elongation, tensile strength or gloss reduction (Kaczmarek 1996a, b). It also results in various harmful effects on the human skin (WHO 1995; Matsumura and Ananthaswamy 2004). Long-term exposure to UV light can result in sunburn, erythema, and acceleration of skin ageing (photoageing) or phototoxic reactions to drugs. Moreover UV radiation can increase risk of skin cancers.

Among other protection measures recommended by dermatologists such as avoiding prolonged exposure on skin, wearing sunglasses, using cosmetics equipped with sunscreen formulations, the use of textile garments is one of the most important and wearing of loose-fitting full-length clothes is recommended by the World Health Organization. Nevertheless, light, bleached, uncoloured or pale-coloured cotton fabrics which are very popular in summer usually do not provide sufficient protection against solar radiation. Protection properties of such textiles can be successfully improved by the use of special auxiliaries which absorb light in the UV-B and UV-A regions (290-400 nm). In order to meet requirements of the industrial practice a typical UV-absorber designed for cellulose fabrics should be water-soluble, show an appropriate affinity to the fibre and lack of added colour. In application terms it could be also regarded as "colourless dye" of the "direct" or "reactive"-type.

UV-absorbers of the "reactive type" contain reactive groups which could form covalent bonds with cellulose after fixation on this fibre and therefore are able to increase protecting properties of modified 
Fig. 1 Reaction of the reactive dichlorotriazine $\mathrm{UV}$ absorber with a cellulose fiber

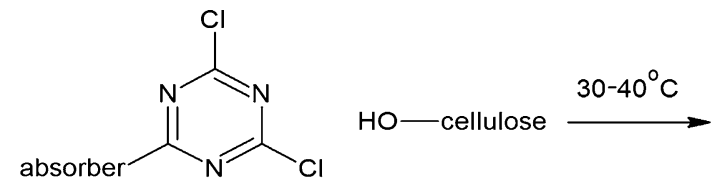

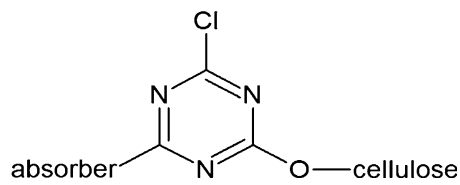

fabrics for prolonged time. Introduction of various reactive groups to $\mathrm{UV}$-absorber molecule makes possible its application at different temperatures.

For example, UV absorbers presented on Fig. 1 containing dichlorotriazine residue could be applied at the temperature range $30-40{ }^{\circ} \mathrm{C}$. More popular in practice bi-functional absorbers shown in Fig. 2 could be used at more elevated temperature range $60-80{ }^{\circ} \mathrm{C}$.

In industrial practice several types of cellulose fibers and also their mixtures with synthetic fibers are used. Among them bamboo viscose, a new regenerated cellulosic fiber has recently evinced quite large interest. The fact, that bamboo plant is a fast-growing renewable resource, is the main advantage of this fabric. However it was reported that untreated bamboo viscose fabrics (similarly as light-weight cotton fabrics) have poor protective properties against UV radiation. Sarkar and Appidi (2009) modified bamboo viscose fabric samples during single-bath application process with the use of commercial UV absorber with or without addition of dye. They reported that good protective properties were reached for UV-absorber concentration $4 \%$ and the dye concentration 2-4\% on the weight of the sample (owf). Fabric samples in which the absorber was applied without the dye have not exhibited satisfactory protection properties even when absorber was used at concentration of $6 \%$ owf.

During our earlier research several types of novel reactive UV absorbers derivatives of symmetrical 1,3,5-triazine designed especially for cellulose fibers were developed (Czajkowski et al. 2005; Czajkowski et al. 2006; Czajkowski and Paluszkiewicz 2008). The aim of present work was to prepare and examine new, cellulose-substantive UV-absorbers characterized by high molar absorption values of the structures presented on Figs. 3, 4 and Table 1. These absorbers were applied on a bamboo viscose tricot and also on other types of the cellulose tricots (cotton, viscose-polyester and viscose-lycra composites). It was expected that these new products would provide good protection properties of modified cellulose fibers at low concentration of used absorbers in application bath.

\section{Experimental}

Materials

Several cellulose-containing tricot samples of comparable weight such as: cotton $\left(177 \mathrm{~g} / \mathrm{m}^{2}\right)$, viscose/

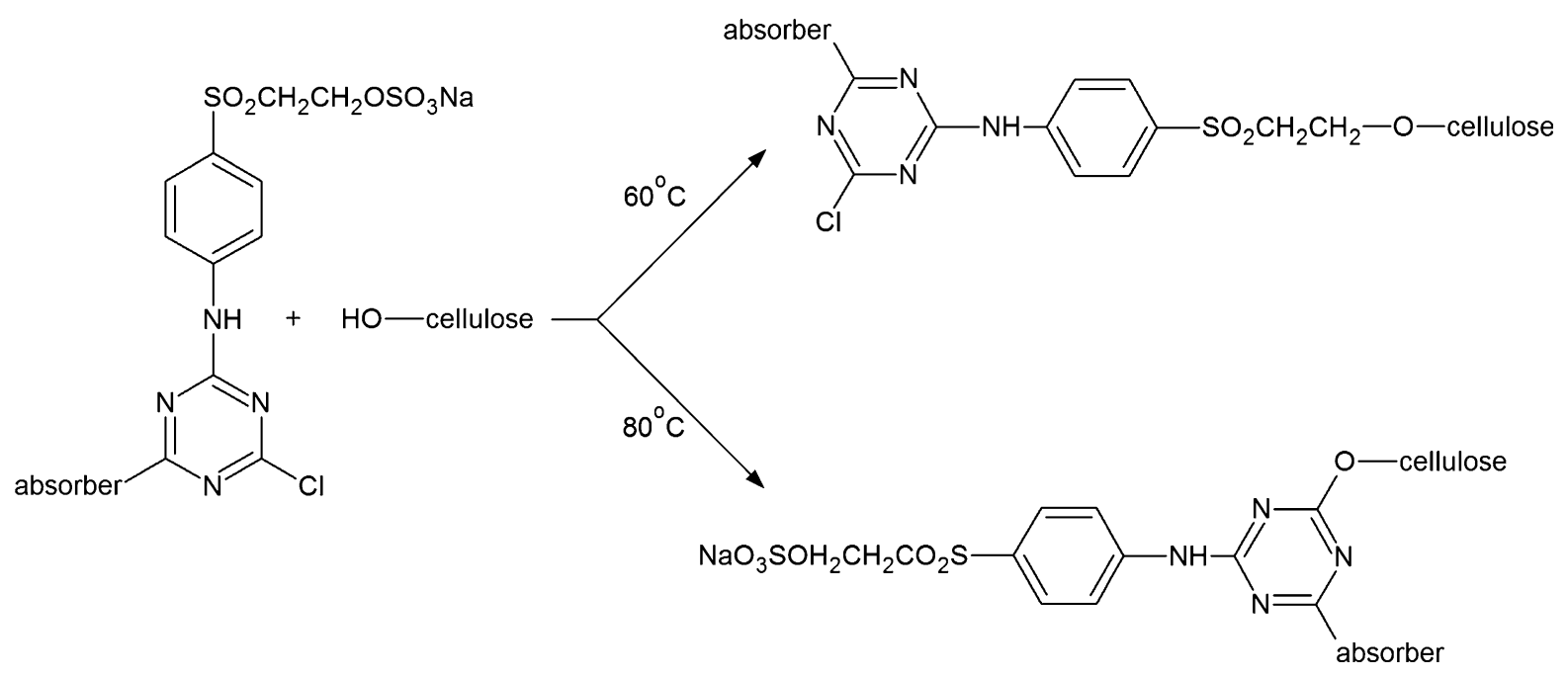

Fig. 2 Reaction of the bi-functional reactive UV absorber with a cellulose fiber 
Fig. 3 Reactive UV-absorbers used in present work, where A-amine
Fig. 4 "Doubledstructure" reactive UV-absorbers used in present work, where B-diamine<smiles>[M]OS([R])([O])c1cc(Nc2nc([Y])nc(Nc3ccc(Nc4nc([R])nc(Cl)n4)c(S(N)(=O)=O)c3)n2)ccc1Nc1nc([R])nc(Cl)n1</smiles><smiles>[R]c1nc(Cl)nc(Nc2ccc(Nc3ncnc(Nc4ccc(Nc5nc([R])nc(Cl)n5)c(S(=O)(=O)O[Na])c4)n3)cc2S(N)(=O)=O)n1</smiles>

polyester 50/50\% (200 g/m $\left.{ }^{2}\right)$, viscose/lycra 94/6\% $\left(230 \mathrm{~g} / \mathrm{m}^{2}\right)$ and bamboo viscose $\left(180 \mathrm{~g} / \mathrm{m}^{2}\right)$ were employed during the experiments. Such textiles are usually applied in practice during manufacturing of light summer clothes.

\section{Characterization of products}

Mass spectra of the synthesized UV-absorbers were taken in a dihydroxybenzoic acid matrix with a Voyager Elite spectrometer using the MALDI (Matrix Assisted Laser Desorption Ionization) technique. Spectrophotometric measurements in the UV-VIS range were taken on a Perkin Elmer Lambda 40 spectrophotometer. The measurements of UV transmittance through the fabric samples were carried out at Textile Research Institute in Lodz with a Jasco V-550 spectrophotometer equipped with an Ulbricht sphere.

Synthesis of intermediates

Intermediate diamines and "tetra"-amines were prepared by three-step synthetic route as it is summarized in Fig. 5 and described in details in the case of intermediate D3.

\section{Preparation of the intermediate "tetra-amine" D3}

$7.53 \mathrm{~g}(0.04 \mathrm{~mol})$ of sodium salt of 2,5-diaminobenzenesulfonic acid was dissolved in water and added to the dispersion of $7.4(0.04 \mathrm{~mol})$ of 2,4,6-trichloro1,3,5-triazine (cyanuric chloride) in ice water with 2 droplets of non-ionic surfactant (Rokanol R9). Condensation process was carried out at $0-5{ }^{\circ} \mathrm{C}$, while maintaining $\mathrm{pH}$ at $6.0-6.5$ by the addition of $10 \%$ $(\mathrm{w} / \mathrm{v})$ aqueous sodium hydroxide. The reaction mixture was stirred for $2 \mathrm{~h}$ while testing the disappearance of 2,5-diaminobenzenesulfonic acid by TLC chromatography (Kieselgel $60 \mathrm{~F}_{254}$ plate; n-butanol: n-propanol: ethyl acetate: water 2:4:1:3 as the eluent). After completing the reaction another portion $6.78 \mathrm{~g}(0.036 \mathrm{~mol})$ of 2.5-diaminobenzenesulfonic acid dissolved in water was added to the mixture and the temperature was increased to $40{ }^{\circ} \mathrm{C}$. The process was carried out at this temperature and $\mathrm{pH}=6.5 \pm 0.2(10 \% \mathrm{NaOH})$ for another 2-3 $\mathrm{h}$. When the end of the reaction had been 
Table 1 UV-absorbers used in present work

\begin{tabular}{llll}
\hline Absorber & Figure & Amine (A) or Diamine (B) & $\mathrm{R}$ \\
\hline A1 & Figure 3 & 3-aminobenzenesulphonic acid (A) & $\mathrm{Cl}$ \\
A1G & & & 4-(sulphatoethyl)sulphonylaniline \\
A2 & Figure 3 & 4-aminobenzoic acid (A) & $\mathrm{Cl}$ \\
A2G & & & 4-(sulphatoethyl)sulphonylaniline \\
A3 & Figure 4 & 4-phenylenediamine (B) & $\mathrm{Cl}$ \\
A3G & & & 4-(sulphatoethyl)sulphonylaniline \\
A4 & Figure 4 & Hydrazine (B) & Cl \\
A4G & & & 4-(sulphatoethyl)sulphonylaniline \\
\hline
\end{tabular}

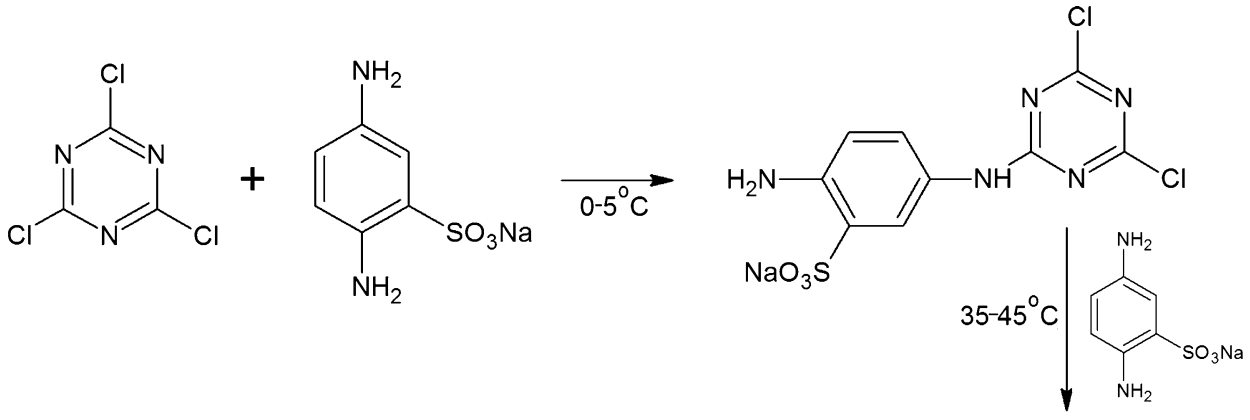<smiles>CS(=O)(=O)c1cc(Nc2nc(Cl)nc(Nc3ccc(N)c(S(C)(=O)=O)c3)n2)ccc1N</smiles>

Diamine $85-90^{\circ} \mathrm{C}$

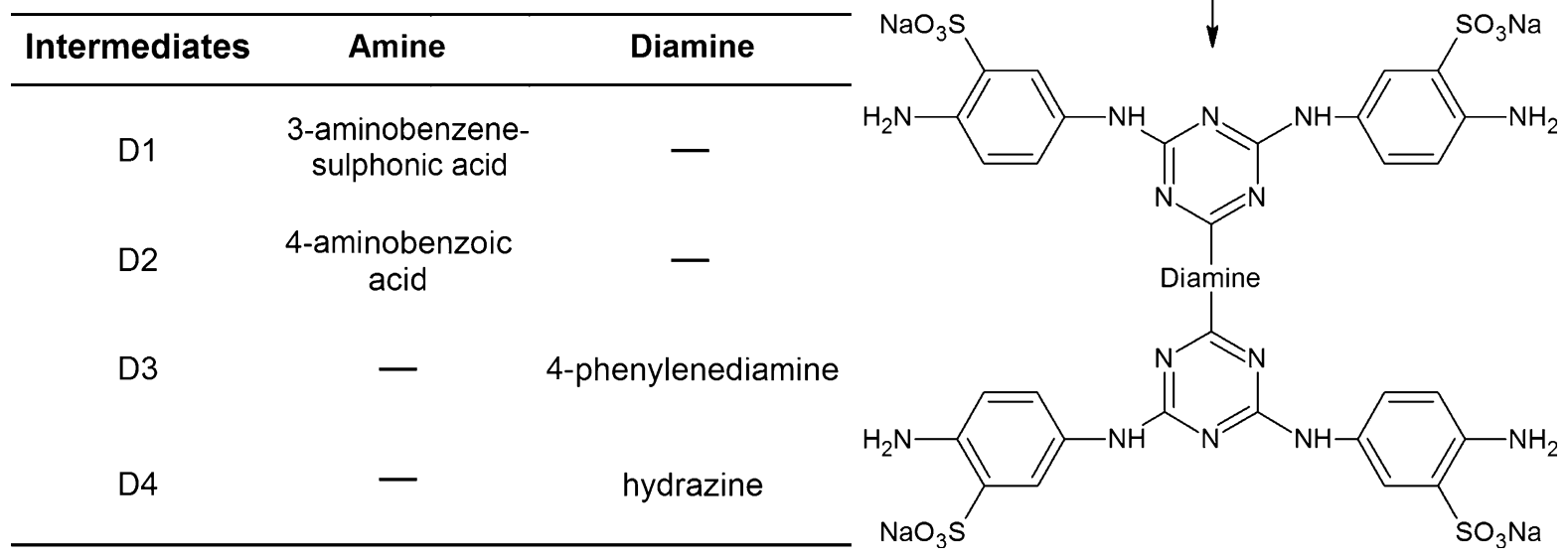

Fig. 5 Synthetic procedures of compounds D1-D4

estimated by TLC analysis, the solution of $2.16 \mathrm{~g}$ (0.02 mol) of 4-phenylenediamine was added. The whole was then heated to $75{ }^{\circ} \mathrm{C}$ and $\mathrm{pH}$ of the mixture was increased from 3.5 to 5.5 by addition of $10 \%$ $\mathrm{NaOH}$ solution. After $30 \mathrm{~min}$ reaction temperature was increased to $85^{\circ} \mathrm{C}$ and $\mathrm{pH}$ to 7.5 . The mixture was 
stirred at these conditions for $2-2.5 \mathrm{~h}$. The completing of the reaction was estimated by TLC. The product was isolated by the addition of $2 \%$ hydrochloric acid, filtered out and washed on the filter by deionized water. The dried product was analyzed by the titration of the sample with $0.1 \mathrm{~N} \mathrm{NaNO}_{2}$ solution.

Synthesis of UV-absorbers

\section{Preparation of absorbers $A 3$ and $A 3 G$}

Solution of $10.11 \mathrm{~g}(0.01 \mathrm{~mol})$ of "tetra-amine D3" in $100 \mathrm{ml}$ of water was slowly added to $7.4(0.04 \mathrm{~mol})$ of cyanuric chloride dispersed in ice-water. The reaction was carried out at $0-5{ }^{\circ} \mathrm{C}$ and $\mathrm{pH}=6.0-6.5(10 \%$ $\mathrm{NaOH})$. Condensation was considered as completed after the disappearance of free amine in the reaction mixture (test on the filter paper with Ehrlich reagent). Reaction mixture in the form of clear light-brown solution was divided to two equal parts.

From the first one absorber A3 (Fig. 4, R = Cl) was separated by salting out with sodium chloride (20\% vol.), filtered, stabilized with phosphate buffer $(\mathrm{pH}=7.0)$ and dried at $35{ }^{\circ} \mathrm{C}$. Finally $20.9 \mathrm{~g}$ of gray powder of approximated purity $47.14 \%$ (estimated by analysis of sodium chloride content by the potentiometric titration with $0.1 \mathrm{~N} \mathrm{AgNO}_{3}$ solution) was obtained. Structure of the product was confirmed by mass spectroscopy during which molecular ions $\mathrm{m} / \mathrm{z}$ $[\mathrm{M}-\mathrm{Na}+2 \mathrm{H}]^{+} \mathrm{m} / \mathrm{z}=1665 ;[\mathrm{M}+3 \mathrm{Na}]^{3+} \mathrm{m} / \mathrm{z}=$ 585 were detected.

To the second part of above mentioned solution of the "tetraamine" D3 $6.07 \mathrm{~g}(0.02 \mathrm{~mol}$ of sodium salt of 4-(ß-sulphatoethyl)sulphonylaniline ("paraester") dissolved in water was added. Condensation process was carried out at the temperature $40{ }^{\circ} \mathrm{C}$ and $\mathrm{pH}=7.0$ $(10 \% \mathrm{NaOH})$. The process was considered as completed after the disappearance of free amine in the reaction mixture (test on a filter paper with the Ehrlich reagent). Absorber A3G was separated by salting out with sodium chloride (20\% vol.), filtered, stabilized with phosphate buffer $(\mathrm{pH}=7.0)$ and dried at $35^{\circ} \mathrm{C}$. Finally $27.3 \mathrm{~g}$ of gray powder of approximated purity $44.22 \%$ (estimated by analysis of sodium chloride content by the potentiometric titration of the sample with $0.1 \mathrm{~N} \mathrm{AgNO}_{3}$ solution) was obtained. Structure of the product was confirmed by mass spectroscopy during which molecular ions $\mathrm{m} / \mathrm{z}[\mathrm{M}+\mathrm{Na}]^{+} \mathrm{m} / \mathrm{z}=2777$; $[\mathrm{M}-4 \mathrm{Na}+8 \mathrm{H}]^{4+} \mathrm{m} / \mathrm{z}=668$ were detected.
Details of the preparation of other absorbers which were obtained in similar way, typical as is used during synthesis of commercial chlorotriazine reactive dyes are given elsewhere (Czajkowski et al. 2010; Czajkowski et al. 2011).

\section{Results and discussion}

Spectral characterisation of the products

Spectroscopic data of the prepared absorbers in the region $250-400 \mathrm{~nm}$ were measured in deionized water (absorber concentration $2 \times 10^{-5} \mathrm{~mol} / \mathrm{dm}^{3}$ ). Results are presented in Table 2 and in Figs. 6, 7.

As it can be seen from the data presented in Table 2 and on Figs. 6, 7 all products absorb light in UV-B range and exhibit similar $\lambda_{\max }$ at $290-300 \mathrm{~nm}$. As it was expected absorbers of the "doubled" structure (A3, A3G, A4 and A4G) are characterized by higher molar absorption coefficients. Moreover, due to the presence of additional diamine bridge, which leads to the increase of interactions between both molecules these compounds, exhibit greater absorbance at longer wavelengths then products without "doubled" structure. At it might be expected introducing 4-(ß-sulphatoethyl) sulphonylaniline residue which includes additional aromatic rings to the absorber molecule (A1G-A4G) results in the further increase of molar absorption coefficient. It can be also noticed that all absorbers have only minor absorbance in the region of $400 \mathrm{~nm}$ so they do not cause yellowing of modified textile materials.

Table 2 Spectral properties of synthesized absorbers

\begin{tabular}{lll}
\hline Absorber & $\lambda_{\max }(\mathrm{nm})$ & Approximate $^{\mathrm{a}} \varepsilon_{\max }\left(\mathrm{dm}^{3} / \mathrm{mol} \mathrm{cm}\right)$ \\
\hline A1 & 286 & $5.5 \times 10^{4}$ \\
A2 & 298 & $3.9 \times 10^{4}$ \\
A3 & 305 & $8.5 \times 10^{4}$ \\
A4 & 299 & $7.9 \times 10^{4}$ \\
A1G & 288 & $8.8 \times 10^{4}$ \\
A2G & 294 & $6.7 \times 10^{4}$ \\
A3G & 291 & $15.5 \times 10^{4}$ \\
A4G & 289 & $13.2 \times 10^{4}$
\end{tabular}

${ }^{a}$ Absorber percentage was estimated by analysis of inorganic salts $(\mathrm{NaCl})$ content in given sample 
Fig. 6 Absorption spectra of absorbers A1, A1G, A2, A2G (water,concentration $2 \times 10^{-5} \mathrm{~mol} / \mathrm{dm}^{3}$, path length $1 \mathrm{~cm}$ )

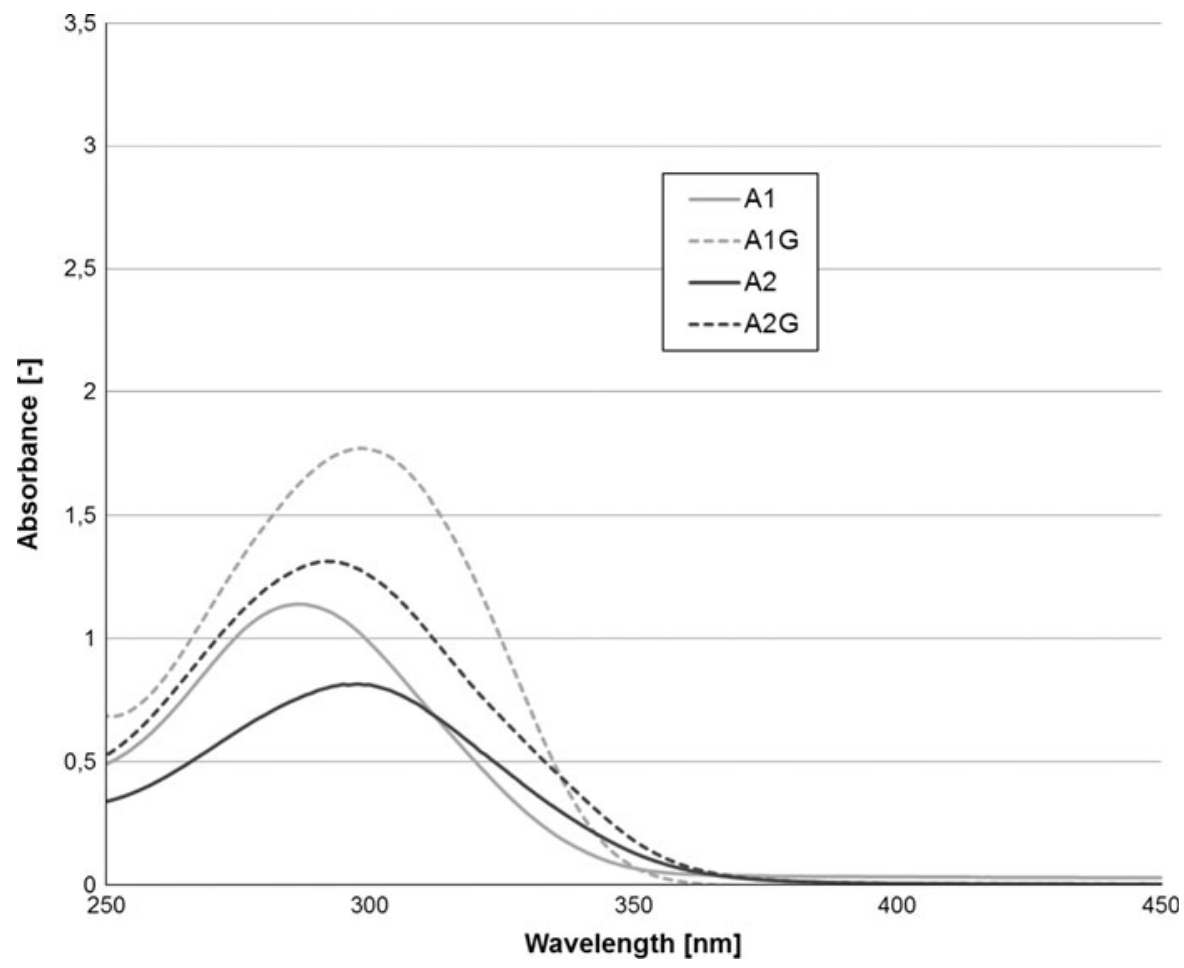

Application tests

All examined UV absorbers were applied by the exhaustion method for used textile substrates at concentrations of 0.5 and $1 \%$ on the weight of used fabrics. Rotec (Roaches) laboratory dyeing machine was used in these experiments. According to actual industrial practice based on environmental considerations during dyeing with the use of reactive dyes in this research 1:15 absorber-bath ratio was applied.

Absorbers A1-A4 were applied similarly as is practiced in the case of dichlorotriazine reactive dyes (temperature $40{ }^{\circ} \mathrm{C} ; \mathrm{Na}_{2} \mathrm{CO}_{3} 20 \mathrm{~g} / \mathrm{dm}^{3} ; \mathrm{Na}_{2} \mathrm{SO}_{4} 40$, 60 and $80 \mathrm{~g} / \mathrm{dm}^{3}$ ). Absorbers A1G-A4G were applied similarly as it is practiced in the case of vinyl sulphone reactive dyes (temperature $60{ }^{\circ} \mathrm{C} ; \mathrm{Na}_{2} \mathrm{CO}_{3} 20 \mathrm{~g} / \mathrm{dm}^{3}$; $\mathrm{Na}_{2} \mathrm{SO}_{4} 40,60$ and $80 \mathrm{~g} / \mathrm{dm}^{3}$ ). After application samples were rinsed twice over $1 \mathrm{~min}$ in separated portions of water at $40{ }^{\circ} \mathrm{C}$ and then oven-dried. The percentage degrees of absorber's exhaustion from application baths were estimated by spectral measurements and calculated according to the formula:

$E=\left(1-\frac{A_{k}}{A_{p}}\right) \times 100 \%$ where $A_{p}$ and $A_{k}$-absorption of the application bath at absorber $\lambda_{\text {max }}$, respectively before and after application test.

Results of these measurements are listed in Table 3.

As it can be noticed all prepared absorbers exhibit good affinity to cellulose fibers. Percentage exhaustion values of all examined products in most of cases exceed $80 \%$. As it was expected the increase of the amount of used electrolyte $\left(\mathrm{Na}_{2} \mathrm{SO}_{4}\right)$ results in the improvement of the exhaustion degree. Absorbers containing 4-(ß-sulphatoethyl)sulphonylaniline residue (A1GA4G) applied at $60{ }^{\circ} \mathrm{C}$ show similar or slightly higher exhaustion degrees than parent "cold type" products applied in $40{ }^{\circ} \mathrm{C}$. It should be also noticed that percentage exhaustion values of the particular absorbers are different in the case of different fabrics. The highest exhaustion values were received in the case of cotton and viscose/polyester tricots (up to $\mathrm{E}=97 \%$ ). Lower (70-80\%) exhaustion degrees even at $0.5 \%$ concentration of the used absorbers may be also noticed in the case of other fabrics.

Measurements of the fabric's protective properties

It was found that application tests at which $80 \mathrm{~g} / \mathrm{dm}^{3}$ $\mathrm{Na}_{2} \mathrm{SO}_{4}$ was present in application baths could provide 
Fig. 7 Absorption spectra of absorbers A3, A3G, A4, A4G (water, concentration $2 \times 10^{-5} \mathrm{~mol} / \mathrm{dm}^{3}$, path length $1 \mathrm{~cm}$ )

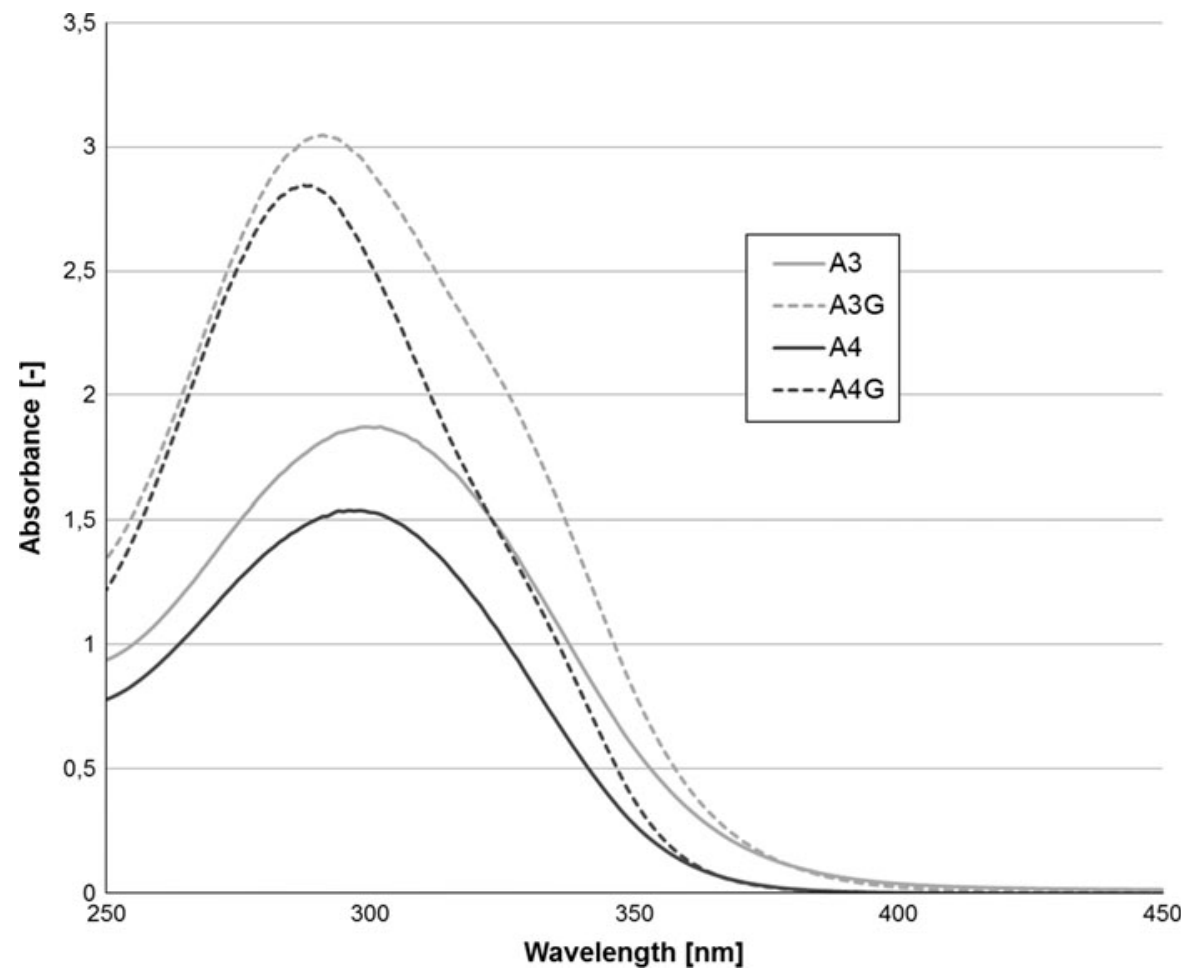

Table 3 Exhaustion degrees of synthesized absorbers on different fabrics as function of different concentrations of used electrolyte

\begin{tabular}{|c|c|c|c|c|c|c|c|c|c|c|c|c|}
\hline \multirow[t]{3}{*}{ Absorber concentration } & \multicolumn{12}{|c|}{$\mathrm{Na}_{2} \mathrm{SO}_{4}\left(\mathrm{~g} / \mathrm{dm}^{3}\right)$} \\
\hline & \multicolumn{3}{|c|}{ Cotton } & \multicolumn{3}{|c|}{ Viscose/poliester } & \multicolumn{3}{|c|}{ Viscose/lycra } & \multicolumn{3}{|c|}{ Bamboo viscose } \\
\hline & 40 & 60 & 80 & 40 & 60 & 80 & 40 & 60 & 80 & 40 & 60 & 80 \\
\hline A1 $0.5 \%$ & 81.2 & 87.9 & 89.5 & 74.4 & 83.6 & 85.8 & 75.5 & 79.8 & 84.9 & 76.5 & 82.1 & 87.2 \\
\hline A1 $1 \%$ & 75.4 & 82.7 & 86.3 & 57.6 & 65.3 & 67.2 & 65.7 & 71.3 & 76.7 & 70.3 & 75.1 & 80.5 \\
\hline A2 $0.5 \%$ & 92.6 & 93.5 & 94.6 & 84.6 & 87.7 & 89.5 & 81.1 & 83.4 & 86.2 & 83.4 & 84.8 & 82.8 \\
\hline A2 $1 \%$ & 90.2 & 92.6 & 93.2 & 73.2 & 80.9 & 80.9 & 73.5 & 74.4 & 77.6 & 73.6 & 76.8 & 73.1 \\
\hline A3 $0.5 \%$ & 94.5 & 95.8 & 97.0 & 83.8 & 91.2 & 94.1 & 68.1 & 73.5 & 76.8 & 63.4 & 75.8 & 77.6 \\
\hline A3 $1 \%$ & 92.2 & 94.8 & 96.5 & 72.4 & 76.5 & 82.5 & 56.1 & 59.0 & 62.0 & 51.7 & 57.3 & 55.8 \\
\hline A4 $0.5 \%$ & 65.0 & 75.4 & 82.3 & 72.7 & 87.2 & 87.8 & 74.3 & 80.6 & 82.9 & 72.7 & 75.7 & 76.5 \\
\hline A4 $1 \%$ & 61.1 & 63.9 & 76.9 & 63.0 & 71.0 & 78.9 & 63.7 & 66.6 & 75.4 & 64.4 & 66.6 & 64.8 \\
\hline A1G $0.5 \%$ & 83.0 & 94.8 & 94.4 & 88.3 & 92.5 & 96.3 & 81.7 & 85.7 & 90.2 & 77.5 & 84.0 & 91.1 \\
\hline A $1 G 1 \%$ & 76.2 & 95.0 & 96.2 & 81.1 & 87.3 & 94.6 & 75.9 & 81.7 & 84.0 & 77.4 & 80.0 & 80.2 \\
\hline A2G $0.5 \%$ & 86 & 95.2 & 95.7 & 83.9 & 89.5 & 93.3 & 73.3 & 79.2 & 83.8 & 74.3 & 78.9 & 85.3 \\
\hline A2G $1 \%$ & 80.4 & 91.8 & 94.4 & 74.0 & 83.8 & 91.6 & 62.1 & 67.5 & 67.7 & 72.5 & 75.5 & 77.1 \\
\hline A $3 \mathrm{G} 0.5 \%$ & 93.2 & 96.0 & 97.1 & 93.3 & 95.9 & 97.5 & 66.8 & 71.9 & 77.3 & 60.7 & 70.1 & 77.2 \\
\hline A3G $1 \%$ & 90.6 & 95.4 & 97.3 & 82.3 & 89.1 & 92.7 & 53.1 & 59.4 & 66.0 & 47.6 & 60.6 & 60.0 \\
\hline A4G $0.5 \%$ & 93.5 & 92.8 & 96.7 & 85.7 & 89.2 & 96.6 & 68.8 & 76.2 & 83.2 & 72.7 & 76.7 & 80.2 \\
\hline A4G $1 \%$ & 90.4 & 90.2 & 95.8 & 78.8 & 86.9 & 94.6 & 58.5 & 67.8 & 77.1 & 58.0 & 68.7 & 72.5 \\
\hline
\end{tabular}


the highest degree of the absorber's exhaustion in the case of all used fabrics. Therefore, modified tricot samples obtained during this experiment were subjected to further investigations during which their protecting properties were determined.

As it is already known Ultraviolet Protection Factor (UPF) is the widely accepted parameter which characterizes protecting properties of textiles. The UPF testing involves exposing a fabric to ultraviolet radiation and measuring how much it is transmitted through the examined sample. The method is given by appropriate European Standard (2007) according to which UPF factor might be calculated according to the formula:

$$
U P F=\frac{\sum_{\lambda=290}^{\lambda=400} E(\lambda) \varepsilon(\lambda) \Delta \lambda}{\sum_{\lambda=290}^{\lambda=400} E(\lambda) T_{i}(\lambda) \varepsilon(\lambda) \Delta \lambda}
$$

where, $E(\lambda)$ is the solar spectral irradiance $\left[\mathrm{W} / \mathrm{m}^{2} \mathrm{~nm}\right]$, $\varepsilon(\lambda)$ the erythema action spectrum, $T_{i}(\lambda)$ the spectral transmittance of sample $i$ at the wavelength $\lambda$ and $\Delta \lambda$ is the interval of wavelength [nm]. The same EU Standard specifies that clothing can bear a "UV-protective" label only if the UPF is higher than 40 and if at the same time UV-A transmission is less than $5 \%$.

Results of UPF measurements of modified tricots' protective properties are graphically presented in Figs. 8 and 9. Data described as "without absorber" were received for samples treated by the "blind bath" containing $\mathrm{Na}_{2} \mathrm{CO}_{3}\left(20 \mathrm{~g} / \mathrm{dm}^{3}\right)$ and $\mathrm{Na}_{2} \mathrm{SO}_{4}(80 \mathrm{~g} /$ $\left.\mathrm{dm}^{3}\right)$.

It can be clearly seen that all of the used absorbers greatly increase UPF values of all examined tricots. Several absorbers especially having doubled-structure (A3, A4, A3G and A4G) provide complete protection properties (UPF> 40) of all used samples even at concentration of $0.5 \%$ on the weight of used fabric. Comparing data presented in Figs. 8 and 9 it can be noticed that increased absorber's concentration in the application bath usually results in increasing of the UPF value. Observed differences are probably the result of various degree of saturation the modified fiber by particular UV-absorbers due to their various affinity to cellulose. The greatest improvement in protective proprieties was achieved for cotton tricot, where the UPF value increased on average almost eight times for $0.5 \%$ application and above ten times for $1 \%$ of absorber's application in the comparison with the unmodified sample (UPF values from $46.1-0.5 \%$ A1 to $141.2-1 \%$ A4G). As it was expected (Reinert et al. 1997) also in present work nature of the used textile material has a significant impact on the value of UPF. Due to its chemical structure polyester fiber absorb radiation in the UV-B region. Its presence in examined viscose-polyester tricot causes that UPF value of untreated sample is much higher than others (UPF = 44). The application of UV absorbers results in the further increase of UPF values from $53(0.5 \% \mathrm{~A} 1)$ to
Fig. 8 Protecting properties of cellulose fabrics after their modification with prepared absorbers used in concentration $0.5 \%$ owf

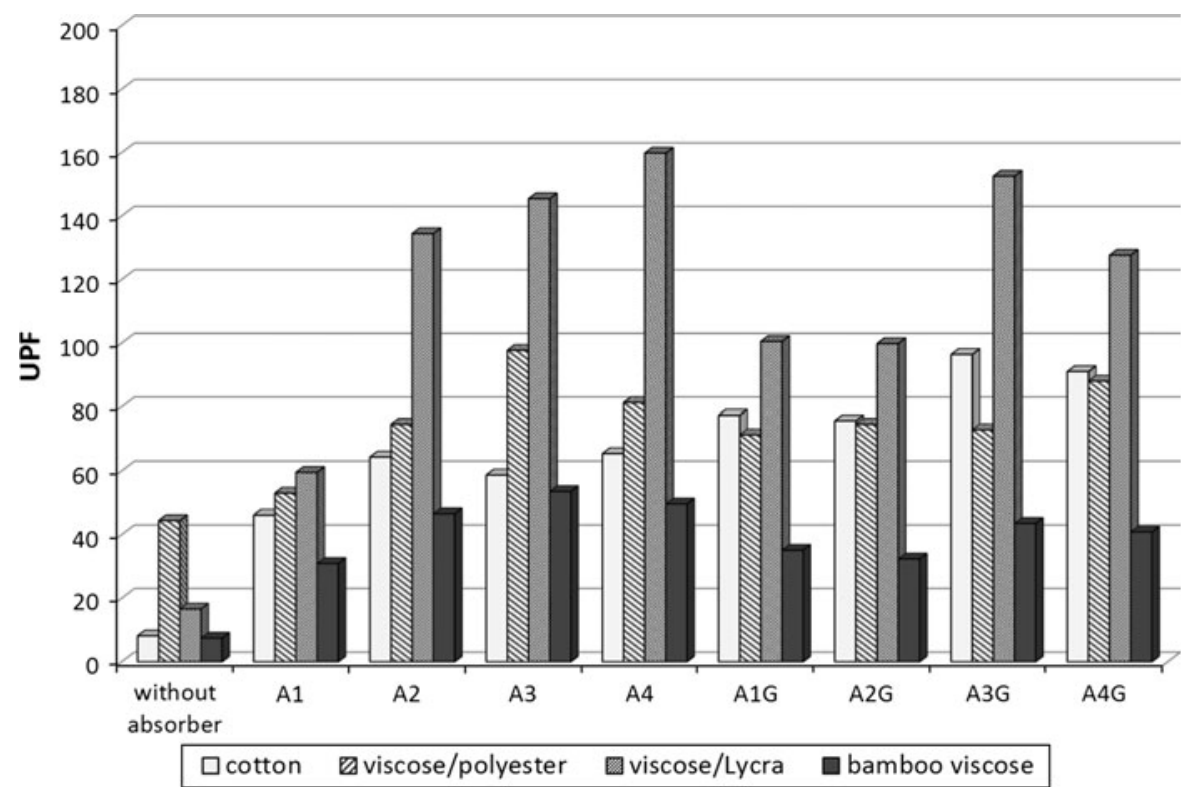


Fig. 9 Protecting properties of cellulose fabrics after their modification with prepared absorbers used in concentration $1 \%$ owf

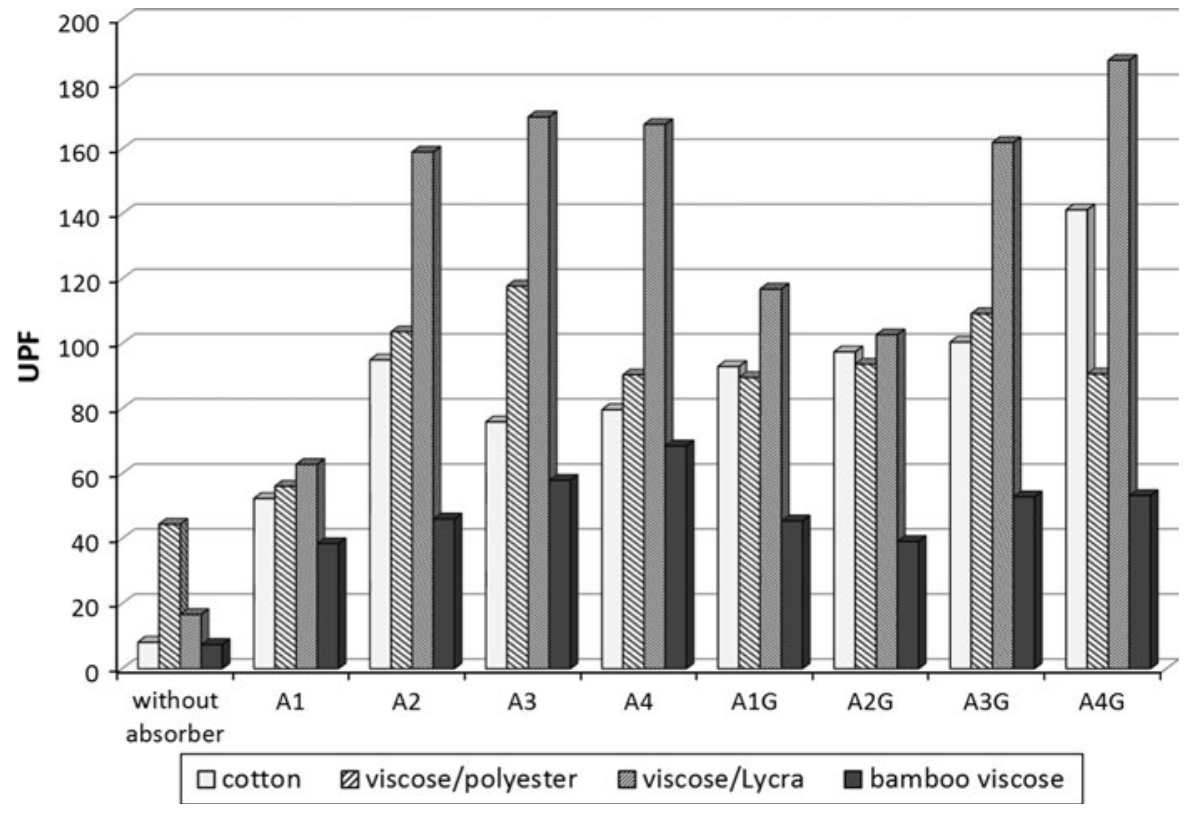

118 (1\% A3). In comparison to the unmodified sample there were achieved improvements in UPF from 20 to even $160 \%$. Nevertheless, as it was mentioned above, materials which UPF is higher than 40 provide sufficient protective properties and in this case the use of UV-absorbers treatment is not necessary.

In the case of bamboo viscose it can be seen that protective properties of modified samples are much lower than other examined samples (UPF values from $30.9-0.5 \%$ A1 to $68.5-1 \%$ A4). This phenomenon has been also observed in other works and may be caused by chemical and structural properties of those textiles such as construction of fabric its thickness and porosity. Rather unexpectedly the highest values of measured UPF exhibits viscose/lycra tricot in which all applied products except A1 give excellent protecting properties. One may suspect that this effect might be partially the result of the presence of free amine groups in polyurethane chain of lycra fiber which are able to form ionic bonds with the absorbers (via their sulphonic groups). Therefore also some hydrolyzed molecules of used absorbers can remind on the fiber after final washing following the application process. It should be also noticed that as it was earlier reported (Algaba and Riva 2002; Algaba et al. 2008; Ghazi et al. 2010; Reinert et al. 1997) also other physical properties of particular fabric are important factors influencing its protective properties and may have influence on observed specific behavior of viscose/lycra composition.

\section{Conclusion}

The results of this work show that all of new synthesized absorbers exhibit good and very good protective properties on all examined samples of cellulose-containing tricots including bamboo viscose. As it was mentioned in earlier work (Sarkar and Appidi 2009), the use of UV-absorber and dye at relative high concentrations in application bath was necessary. In present work some of developed reactive UV-absorbers despite their medium affinity to examined bamboo-viscose tricot provided good protective properties even when used in $0.5 \%$ concentration per weight of the fabric. In the case of other examined fabrics all used UV absorbers gave excellent results. The presence of synthetic fibers which exhibit own absorbance of UV-radiation (lycra, polyester) also significant improves the barrier properties of textiles.

Acknowledgments The authors wish to thank Marcin Kudzin from Textile Research Institute in Lodz Poland for his help in measurements of UPF values. This publication was partially prepared within the key project - PO IG no. 01.03.01-00-006/08 co-financed from the funds of European Regional Development 
Fund within the framework of the Operational Program Innovative Economy.

Open Access This article is distributed under the terms of the Creative Commons Attribution License which permits any use, distribution, and reproduction in any medium, provided the original author(s) and the source are credited.

\section{References}

Algaba I, Riva A (2002) In vitro measurement of the ultraviolet protection factor of apparel textiles. Color Technol 118: $52-58$

Algaba I, Pepió M, Riva A (2008) Correlation between the ultraviolet protection factor and the weight and thickness of undyed cellulosic woven fabrics. Fibres Text East Eur 16(1):85-89

Czajkowski W, Paluszkiewicz J (2008) Synthesis of bifunctional monochlorotriazine reactive dyes increasing UVprotection properties of cotton fabrics. Fibres Text East Eur 16(5):122-126

Czajkowski W, Paluszkiewicz J, Stolarski R (2005) Polish patent application P-374411

Czajkowski W, Paluszkiewicz J, Stolarski R, Kaźmierska M, Grzesiak E (2006) Synthesis of reactive UV absorbers derivatives of monochlorotriazine for improvement protecting properties of cellulose fabrics. Dyes Pigment 71: 224-230
Czajkowski W, Paluszkiewicz J, Mamnicka J, Stolarski R (2010) Polish patent application P-390435

Czajkowski W, Mamnicka J, Lewartowska J, Sojka-Ledakowicz J (2011) Polish patent application P-394616

European Standards; PN-EN 13758-1:2007 Textiles-Solar UV protective properties-Part 1: method of test for apparel fabrics; PN-EN 13758-2:2007 Textiles-Solar UV protective properties-Part 2: classification and marking of apparel

Ghazi S, Couteau C, Coiffard LJM (2010) What level of protection can be obtained using sun protective clothing. Int $\mathrm{J}$ Pharm 397:144-146

Kaczmarek H (1996a) Changes to polymer morphology caused by UV irradiation: 1 surface damage. Polymer 37(2):189-194

Kaczmarek H (1996b) Changes of polymer morphology caused by uv irradiation: 2 surface destruction of polymer blends. Polymer 37(4):547-553

Matsumura Y, Ananthaswamy HN (2004) Toxic effects of ultraviolet radiation on the skin. Toxicol Appl Pharm 195: 298-308

Reinert G, Fuso F, Hilfiker R, Schmidt E (1997) UV-Protecting properties of textile fabrics and their improvement. Text Chem Color 29:36-43

Sarkar AK, Appidi S (2009) Single bath process for imparting antimicrobial activity and ultraviolet protective property to bamboo viscose fabric. Cellulose 16(5):923-928

World Health Organization, Environmental Health Criteria 160 WHO/EHG/95.16. http://www.who.int/uv/publica tions/UVEHeffects.pdf. Accessed 03 Feb 2012 